\title{
Recent Empirical Analysis on Public Service Companies Transparency: Case of Italy
}

\author{
Blerina Banushi, PhD Candidate \\ University of Tirana, Albania \\ Maurizio Rija, Prof. \\ University of Calabria, Italy
}

URL:http://dx.doi.org/10.19044/esj.2019.v15n1p46

\begin{abstract}
In recent years the role of public services has acquired increasing relevance on account of the growing awareness of the importance of such services in the social and economic development of a country. This is determined primarily by the demand for quality and good value (for money) by an ever more sensitive public (which has brought under analysis the methods of traditional service providers, no longer seen as able to respond efficiently to the changing and diversified needs of society), the need to conform to EU directives and the effects of liberalization and from an internal perspective of the industrial and institutional reorganization of the public sector.

After a description of the theoretical background, the present study is aimed to analyse the transparency of the public service companies listed on the Italian Stock Exhange. Given the fundamental role played by these companies, this sudy investigates the levels of total and partial disclosure and identifies the successful elements as well as those areas where improvements could be made. In particular, for the research conducted, an attempt was made to adopt an index capable of showing a measure of the quality of transparency undertaken by the case-study companies in the FTSE Italy Public Services Sector. In order to meet this aim, the work is based on an index of "corporate e-governance" used by Gandia.

Based on the variables considered in the analysis of the websites, no company provides total disclosure with maximum values; the company which offers total disclosure with the highest value is Enel (9.52) while Ergycapital has the lowest value (3.14). Research thus shows that, it is necessary to improve the quality of disclosure of the public utilities companies listed on the Italian Stock Exchange.
\end{abstract}

Keywords: Public utilities, disclosure, governance, investors 


\section{Introduction:}

Public utility services are characterized by a demand and a singularly identifiable use; their cession can occur in the form of typical exchange, or with the formation of a price. They come under the category of both public services and network services (Stiglitz, 2003).

The public services sector presents some peculiarities and multiple types of operators within the sector according to a geographical, business or ownership perspective (Chan and Watson, 2011). The geographical perspective, where it is possible to identify a local, national and international dimension, refers to the territorial extension that marks business operability. The local dimension characterizes the majority of the companies operating in the public services sector in the Italian market has been historically dominated by realities that are closely linked to the territory, such as the ex-municipalized companies that continue to serve their areas of origin.

The national dimension regards those companies, often publicly owned, that have developed their own operability at the level of a single country. They are former national monopolies that have consolidated their presence in the reference countries within the political direction set by respective governments; Enel and Gaz de France are two such examples in the energy sector. The international dimension finds its reasons in a greater contractual power in procurement, greater financial capacity, strategic and operative synergy attainable in the execution of fundamental activities and market opportunities. In the latter one can think of the German case E.On or Endesa of Spain that acquired market shares where liberalization processes had effectively occurred and where the possibility of generating profits resulted as being more significant.

The perspective of business refers to the strategic choices, or rather the portfolio offer (mono-utility, bi-utility, multi-utility, multi-service), executed by companies regarding the nature of services offered. Mono-utility companies are concentrated in a sole sector by means of a strategic focalization, which is often accompanied by research of territorial expansion and integration either before or after, whilst remaining ever careful to not go beyond the pre-chosen industry. Bi-utility companies are characterized by their choice to operate in two activity sectors, often because of the objective synergies that can be generated by such a pairing. An example is provided by the joint presence of gas and hydro (due to synergies deriving from network management) or between electrical energy and gas (due to the synergies attainable in procurement where the combined cycle for energy production uses natural gas). Instead, multi-utility companies are characterized by the connected supply of three or more services considered here. The strength of the companies examined lies in their link with the territory and their aim of providing more network public services (electrical, hydro, 
telecommunications, etc.) using technical and commercial synergies. Instead, multi-service companies follow the logic of the territorial presence and availability of a consolidated client portfolio using the incentive of traditional business as stimuli for new activities which lie outside the area of utilities. Examples of this type of model are the spread of credit cards, the provision of insurance policies, telecommunications services, but also the management of green spaces, facility management, and the management of infrastructures for free time.

The ownership perspective refers to the economic subject that governs the company, in such a context there can be companies with a totally public capital, with public majority, publicly controlled listed on the stock exchange, with a private majority and totally private (Bianchi et al. 2011).

In the context of public utilities companies (which often have activities in sectors that were formerly monopolies and of considerable interest, not only from economic but also of social relevance) corporate disclosure assumes a particular connotation which should, amongst other things, facilitate respect for legality, transparency of administrative actions and the interests of the collectivity which the company serves.

The listed public utility company must seek to guarantee the adoption of practices in order to ensure protection of the multiple interests that are in it, precisely due to its particular ownership structure (Maronti and Iuliano, 2012).

With listing on the stock exchange, public utility companies, which operate in the fields of electrical energy, natural gas, environmental services and water services, encounter the opening of capital to other subjects whose interests are multiple and, at times, different. Thus, the existing relation between ownership and control of the company is significantly modified. In particular, other subjects characterized by different motivational logics such as the development of business and business idea, the valorization and growth of investment value and so on join the public subject.

\section{I.:}

The internet currently represents one of the most powerful means that companies can employ to provide information and manage their relations with their stakeholders. The web offers businesses the possibility of reaching a vast array of users and, therefore, of providing them with financial, operative and governance information without temporal or spatial limitations.

In accordance with that held by Cucuzza and Cherian (2001) e-business instruments represent a new path for communicating a large amount of information in an environment in which information flows are continuously updated. This research arises from the discovery of various studies (Quagli, 2002) that highlight the importance of the internet as a communication channel between companies and their investors and of others (Craven and Marston, 
1999; Xiao et al. 2004; Debreceny et al. 2002; Debreceny and Rahman, 2005; Bonson and Escobar, 2006; Kahn, 2007) which have concentrated on the use of the internet as a means of communicating financial data.

Financial communication through the internet presents different aspects compared to traditional presentations on paper, among which the topicality of the presentations and the process of information access (Kahn, 2007) are to be remembered. The application and development of internet based corporate governance disclosure facilitates communication between companies and investors; it reduces the distribution costs of documents and improves the temporal periodicity of information, facilitates the exercising of shareholder voting rights in listed companies, democratizes the process of access to company information and improves the credibility of corporate governance practices (Gandia, 2008).

The aim of the research described hereafter, referring to the works of Gandia (2008) and Khan (2007), was that of examining the presence of some characteristics in disclosure by companies operating in the public utilities sector which are listed on the Italian Stock Exchange, on their websites in February 2017.

In particular, for the research conducted, an attempt was made to adopt an index capable of showing a measure of the quality of transparency undertaken by the companies. In order to meet this aim, the work is based on an index of "corporate e-governance" used by Gandia. The author then analyzed the three groups of characteristics to be found on the sites and then proceeded to calculate a total transparency index (Gandia, 2008). Specifically, the general characteristics needed are the usability of the website and financial disclosure, disclosure regarding the administrative body and shareholders and disclosure regarding other elements of corporate governance. After having collected economic and financial data, governance reports and other information, a comparative analysis was conducted on the following companies in the FTSE Italy Public Services Sector: A2a, Acea, Acsm-Agam, Alerion Cleanpower, Ascopiave, Edison Rsp, Enel, Erg, Ergycapital, Falck Renewables, Hera, Iren, Italgas, K.R.Energy, Snam, Terna (National Electric Grid) and Ternienergia. The characteristics sought are indicated in the Tables $\mathrm{A}, \mathrm{B}$ and $\mathrm{C}$.

Table A - First group of features

\begin{tabular}{|l|l|}
\hline \multicolumn{1}{|c|}{ A) USABILITY OF WEBSITE AND FINANCIAL DISCLOSURE } \\
\hline \multicolumn{1}{|c|}{ Feature } & \multicolumn{1}{c|}{ We verified whether the website: } \\
\hline $\begin{array}{l}\text { 1) Corporate governance } \\
\text { section }\end{array}$ & $\begin{array}{l}\text { Contained a section exclusively dedicated to corporate } \\
\text { governance }\end{array}$ \\
\hline $\begin{array}{l}\text { 2) Documents in various } \\
\text { formats }\end{array}$ & $\begin{array}{l}\text { Offered the possibility of downloading financial } \\
\text { documents (financial statements, presentations, etc.) } \\
\text { in various formats (excel spreadsheets, interactive } \\
\text { pdfs) }\end{array}$ \\
\hline
\end{tabular}




\begin{tabular}{|l|l|}
\hline $\begin{array}{l}\text { 3) Multimedia } \\
\text { presentations }\end{array}$ & $\begin{array}{l}\text { Supported the presentation of financial data in } \\
\text { multimedia format (java, flash, etc.) }\end{array}$ \\
\hline 4) Contacts section & $\begin{array}{l}\text { Presented a section containing email addresses or } \\
\text { other means of communicating with the company }\end{array}$ \\
\hline 5) E-mail alert & $\begin{array}{l}\text { Offered users the opportunity to receive information } \\
\text { regarding news on economic financial and governance } \\
\text { data }\end{array}$ \\
\hline 6) English version & Offered an English version \\
\hline $\begin{array}{l}\text { 7) Event calendar } \\
\text { (updated) }\end{array}$ & $\begin{array}{l}\text { Presented an updated calendar with future company } \\
\text { events }\end{array}$ \\
\hline $\begin{array}{l}\text { 8) Annual financial } \\
\text { statements }\end{array}$ & $\begin{array}{l}\text { Offered the possibility of downloading the financial } \\
\text { statement in pdf }\end{array}$ \\
\hline 9) Indices & $\begin{array}{l}\text { Offered the possibility of consulting the economic } \\
\text { financial indices }\end{array}$ \\
\hline $\begin{array}{l}\text { 10) Financial statement } \\
\text { archive }\end{array}$ & Contained previous years' financial statements \\
\hline
\end{tabular}

Table B - Second group of features

\begin{tabular}{|c|c|}
\hline \multicolumn{2}{|c|}{$\begin{array}{l}\text { B) DISCLOSURE REGARDING THE ADMINISTRATIVE BODY AND } \\
\text { SHAREHOLDERS }\end{array}$} \\
\hline Feature & We verified whether the website: \\
\hline $\begin{array}{l}\text { 1) Members of the } \\
\text { administrative body }\end{array}$ & $\begin{array}{l}\text { Reported the names of the components of the } \\
\text { executive body in html format }\end{array}$ \\
\hline $\begin{array}{l}\text { 2) Structure of the } \\
\text { administrative body }\end{array}$ & $\begin{array}{l}\text { Contained an indication of the structure of the } \\
\text { administrative body (president, chief executive } \\
\text { officer, executive, non-executive and independent } \\
\text { directors) in html }\end{array}$ \\
\hline $\begin{array}{l}\text { 3) Curricula of the members } \\
\text { of the administrative body }\end{array}$ & $\begin{array}{l}\text { Published the curricula of the members of the } \\
\text { administrative body }\end{array}$ \\
\hline $\begin{array}{l}\text { 4) Functioning of the } \\
\text { administrative body }\end{array}$ & $\begin{array}{l}\text { Published the rules regarding organization and } \\
\text { functioning of the administrative body }\end{array}$ \\
\hline $\begin{array}{l}\text { 5) Functioning } \\
\text { committees within the } \\
\text { administrative body }\end{array}$ & $\begin{array}{l}\text { Published the rules regarding organization and } \\
\text { functioning of the committees within the } \\
\text { administrative body }\end{array}$ \\
\hline 6) Remuneration policies & $\begin{array}{l}\text { the remuneration mechanisms of members } \\
\text { ministrative body }\end{array}$ \\
\hline $\begin{array}{l}\text { 7) Emoluments of } \begin{array}{r}\text { the } \\
\text { body }\end{array} \\
\text { administrative } \\
\text { members }\end{array}$ & $\begin{array}{l}\text { Indicated the emoluments paid to members of the } \\
\text { administrative body }\end{array}$ \\
\hline 8) Election procedure & $\begin{array}{l}\text { The election procedures of members of the } \\
\text { administrative body were present }\end{array}$ \\
\hline 9) Major shareholders & $\begin{array}{l}\text { The distribution of shares between major shareholders } \\
\text { were indicated }\end{array}$ \\
\hline $\begin{array}{l}\text { 10) Share ownership of } \\
\text { administrative body } \\
\text { members }\end{array}$ & $\begin{array}{l}\text { The shares held by members of the administrative } \\
\text { body were indicated }\end{array}$ \\
\hline
\end{tabular}


Table C - Third group of features

C) DISCLOSURE ON OTHER ELEMENTS OF CORPORATE GOVERNANCE

\begin{tabular}{|l|l|}
\hline \multicolumn{1}{|c|}{ Feature } & \multicolumn{1}{c|}{ We verified whether the website: } \\
\hline 1) Rules and procedures & Contained the rules and procedures \\
\hline 2) Company statute & Contained the company statute \\
\hline 3) Ethical code & $\begin{array}{l}\text { Contained the ethical code implemented by the } \\
\text { company }\end{array}$ \\
\hline 4) Sustainability report & Presented the sustainability report of the company \\
\hline $\begin{array}{l}\text { 5) Information on the board } \\
\text { of statutory auditors }\end{array}$ & $\begin{array}{l}\text { Information on the board of statutory auditors were } \\
\text { present }\end{array}$ \\
\hline 6) Analyst coverage & A dedicated company analysis page \\
\hline 7) Auditing firm & The auditing firm was indicated \\
\hline
\end{tabular}

The index was calculated taking into consideration the variables of the preceding tables. If a company provides the information in question it will be awarded one point, otherwise it will receive zero points. First, a calculation of partial points was made and then a calculation of the total index. The partial indices were calculated in the following manner:

$$
\begin{array}{r}
I A=\left(\frac{\text { score } \cdot \text { obtained } \cdot \text { by } \cdot \text { the } \cdot \text { company } \cdot \text { in } \cdot \text { table } \cdot A}{\text { maximum } \cdot \text { obtainable } \cdot \text { score } \cdot \text { in } \cdot \text { table } \cdot A}\right) \times 10 \\
I B=\left(\frac{\text { score } \cdot \text { obtained } \cdot \text { by } \cdot \text { the } \cdot \text { company } \cdot \text { in } \cdot \text { table } \cdot B}{\text { maximum } \cdot \text { obtainable } \cdot \text { score } \cdot \text { in } \cdot \text { table } \cdot B}\right) \times \cdot 10 \\
I C=\left(\frac{\text { score } \cdot \text { obtained } \cdot \text { by } \cdot \text { the } \cdot \text { company } \cdot \text { in } \cdot \text { table } \cdot A}{\text { maximum } \cdot \text { obtainable } \cdot \text { score } \cdot \text { in } \cdot \text { table } \cdot C}\right) \times \cdot 7
\end{array}
$$

The total index is given by the following formula:

$$
I T=\left[\left(\frac{I A+I B+I C}{\text { number } \cdot \text { of } \cdot \text { partial } \cdot \text { indices }}\right)\right] \times \cdot 10
$$

For their construction, both the partial indices and the total index vary by a minimum of zero and by a maximum of ten.

The following information was acquired in the month of February 2017 on the websites of the companies (A2A, Acea, Acsm-Agam, Alerion Clean Power, Ascopiave, Edison, Enel, Erg, ErgyCapital, Falck Renewables, Hera, Iren, Italgas, Krenegy, Snam, Terna, TerniEnergia).

The results obtained from the analysis are reported in the following Tables D and E. 
Table D - Partial summary of obtained results

\begin{tabular}{|c|c|c|c|c|c|c|c|c|c|c|c|c|c|c|c|c|c|}
\hline \multicolumn{18}{|c|}{$\begin{array}{l}\text { A) USABILITY OF WEBSITE AND FINANCIAL } \\
\text { DISCLOSURE }\end{array}$} \\
\hline \begin{tabular}{l|l}
$\mathbf{F}$ & \\
$\mathbf{E}$ & $\mathbf{Z}$ \\
$\mathbf{A}$ & $\mathbf{\Omega}$ \\
$\mathbf{T}$ & $\mathbf{z}$ \\
$\mathbf{U}$ & $\mathbf{0}$ \\
$\mathbf{R}$ & $\mathbf{U}$ \\
$\mathbf{E}$ &
\end{tabular} & $\underset{Z}{3}$ & 选 & $\sum_{\substack{\infty \\
\infty}}^{\sum_{4}^{1}}$ & 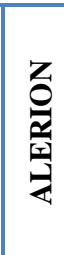 & 焉 & 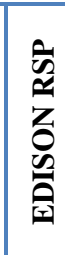 & 国 & 㫕 & 造 & 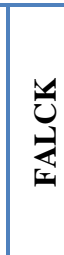 & $\frac{\pi}{\underline{x}}$ & 罕 & 式 & 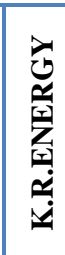 & 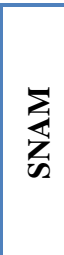 & 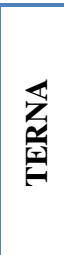 & 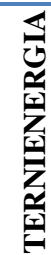 \\
\hline $\begin{array}{l}\text { 1) } \\
\text { Corpor } \\
\text { ate } \\
\text { governa } \\
\text { nce } \\
\text { section } \\
\end{array}$ & $\begin{array}{l}\mathrm{Y} \\
\mathrm{ES}\end{array}$ & $\begin{array}{l}\mathrm{Y} \\
\mathrm{ES}\end{array}$ & $\begin{array}{l}\text { Y } \\
\text { ES }\end{array}$ & $\begin{array}{l}\text { Y } \\
\text { ES }\end{array}$ & $\begin{array}{l}\text { Y } \\
\text { ES }\end{array}$ & $\begin{array}{l}\text { Y } \\
\text { ES }\end{array}$ & $\begin{array}{l}\text { Y } \\
\text { ES }\end{array}$ & $\begin{array}{l}\text { Y } \\
\text { ES }\end{array}$ & $\begin{array}{l}\text { Y } \\
\text { E } \\
\text { S }\end{array}$ & $\begin{array}{l}\mathrm{Y} \\
\mathrm{ES}\end{array}$ & $\begin{array}{l}\mathrm{Y} \\
\mathrm{ES}\end{array}$ & $\begin{array}{l}\mathrm{Y} \\
\mathrm{ES}\end{array}$ & $\begin{array}{l}\text { Y } \\
\text { E } \\
\text { S }\end{array}$ & $\begin{array}{l}\text { Y } \\
\text { ES }\end{array}$ & $\begin{array}{l}\mathrm{Y} \\
\mathrm{ES}\end{array}$ & $\begin{array}{l}\text { Y } \\
\text { ES }\end{array}$ & $\begin{array}{l}\text { Y } \\
\text { ES }\end{array}$ \\
\hline $\begin{array}{l}\text { 2) } \\
\text { Docume } \\
\text { nts in } \\
\text { various } \\
\text { formats }\end{array}$ & $\begin{array}{l}\text { Y } \\
\text { ES }\end{array}$ & $\begin{array}{l}\mathrm{Y} \\
\mathrm{ES}\end{array}$ & \begin{tabular}{|l} 
Y \\
ES
\end{tabular} & $\begin{array}{l}\text { Y } \\
\text { ES }\end{array}$ & $\begin{array}{l}\text { Y } \\
\text { ES }\end{array}$ & $\begin{array}{l}\text { Y } \\
\text { ES }\end{array}$ & $\begin{array}{l}\text { Y } \\
\text { ES }\end{array}$ & $\begin{array}{l}\mathrm{Y} \\
\mathrm{ES}\end{array}$ & $\begin{array}{l}\mathrm{Y} \\
\mathrm{E} \\
\mathrm{S}\end{array}$ & $\begin{array}{l}\text { Y } \\
\text { ES }\end{array}$ & $\begin{array}{l}\text { Y } \\
\text { ES }\end{array}$ & $\begin{array}{l}\text { Y } \\
\text { ES }\end{array}$ & $\begin{array}{l}\text { Y } \\
\text { E } \\
\text { S }\end{array}$ & $\begin{array}{l}\mathrm{N} \\
\mathrm{O}\end{array}$ & $\begin{array}{l}\text { Y } \\
\text { ES }\end{array}$ & $\begin{array}{l}\text { Y } \\
\text { ES }\end{array}$ & $\begin{array}{l}\text { Y } \\
\text { ES }\end{array}$ \\
\hline $\begin{array}{l}\text { 3) } \\
\text { Multim } \\
\text { edia } \\
\text { present } \\
\text { ations }\end{array}$ & $\begin{array}{l}\text { Y } \\
\text { ES }\end{array}$ & \begin{tabular}{|l} 
Y \\
ES
\end{tabular} & $\begin{array}{l}\text { Y } \\
\text { ES }\end{array}$ & $\begin{array}{l}\mathrm{N} \\
\mathrm{O}\end{array}$ & $\begin{array}{l}\text { Y } \\
\text { ES }\end{array}$ & $\begin{array}{l}\text { Y } \\
\text { ES }\end{array}$ & $\begin{array}{l}\text { Y } \\
\text { ES }\end{array}$ & $\begin{array}{l}\mathrm{Y} \\
\mathrm{ES}\end{array}$ & $\begin{array}{l}\mathrm{N} \\
\mathrm{O}\end{array}$ & $\begin{array}{l}\text { Y } \\
\text { ES }\end{array}$ & $\begin{array}{l}\mathrm{Y} \\
\mathrm{ES}\end{array}$ & $\begin{array}{l}\text { Y } \\
\text { ES }\end{array}$ & $\begin{array}{l}\text { Y } \\
\text { E } \\
\text { S }\end{array}$ & $\begin{array}{l}\mathrm{N} \\
\mathrm{O}\end{array}$ & $\begin{array}{l}\text { Y } \\
\text { ES }\end{array}$ & $\begin{array}{l}\text { Y } \\
\text { ES }\end{array}$ & $\begin{array}{l}\text { Y } \\
\text { ES }\end{array}$ \\
\hline $\begin{array}{l}\text { 4) } \\
\text { Contact } \\
\text { s } \\
\text { section }\end{array}$ & $\begin{array}{l}\text { Y } \\
\text { ES }\end{array}$ & $\begin{array}{l}\text { Y } \\
\text { ES }\end{array}$ & $\begin{array}{l}\text { Y } \\
\text { ES }\end{array}$ & $\begin{array}{l}\text { Y } \\
\text { ES }\end{array}$ & $\begin{array}{l}\mathrm{Y} \\
\mathrm{ES}\end{array}$ & $\begin{array}{l}\text { Y } \\
\text { ES }\end{array}$ & $\begin{array}{l}\text { Y } \\
\text { ES }\end{array}$ & $\begin{array}{l}\mathrm{Y} \\
\mathrm{ES}\end{array}$ & $\begin{array}{l}\mathrm{Y} \\
\mathrm{E} \\
\mathrm{S}\end{array}$ & $\begin{array}{l}\text { Y } \\
\text { ES }\end{array}$ & $\begin{array}{l}\text { Y } \\
\text { ES }\end{array}$ & $\begin{array}{l}\text { Y } \\
\text { ES }\end{array}$ & $\begin{array}{l}\text { Y } \\
\text { E } \\
\text { S }\end{array}$ & $\begin{array}{l}\text { Y } \\
\text { ES }\end{array}$ & $\begin{array}{l}\mathrm{Y} \\
\mathrm{ES}\end{array}$ & $\begin{array}{l}\text { Y } \\
\text { ES }\end{array}$ & $\begin{array}{l}\text { Y } \\
\text { ES }\end{array}$ \\
\hline $\begin{array}{l}\text { 5) } \mathrm{E}- \\
\text { mail } \\
\text { alert }\end{array}$ & $\begin{array}{l}\mathrm{Y} \\
\mathrm{ES}\end{array}$ & $\begin{array}{l}\mathrm{N} \\
\mathrm{O}\end{array}$ & $\begin{array}{l}\mathrm{N} \\
\mathrm{O}\end{array}$ & $\begin{array}{l}\mathrm{N} \\
\mathrm{O}\end{array}$ & $\begin{array}{l}\mathrm{N} \\
\mathrm{O}\end{array}$ & $\begin{array}{l}\mathrm{N} \\
\mathrm{O}\end{array}$ & $\begin{array}{l}\text { Y } \\
\text { ES }\end{array}$ & $\begin{array}{l}\mathrm{Y} \\
\mathrm{ES}\end{array}$ & $\begin{array}{l}\mathrm{N} \\
\mathrm{O}\end{array}$ & $\begin{array}{l}\mathrm{N} \\
\mathrm{O}\end{array}$ & $\begin{array}{l}\text { Y } \\
\text { ES }\end{array}$ & $\begin{array}{l}\mathrm{N} \\
\mathrm{O}\end{array}$ & $\begin{array}{l}\mathrm{N} \\
\mathrm{O}\end{array}$ & $\begin{array}{l}\mathrm{N} \\
\mathrm{O}\end{array}$ & $\begin{array}{l}\text { Y } \\
\text { ES }\end{array}$ & $\begin{array}{l}\mathrm{N} \\
\mathrm{O}\end{array}$ & $\begin{array}{l}\mathrm{N} \\
\mathrm{O}\end{array}$ \\
\hline $\begin{array}{l}\text { 6) } \\
\text { English } \\
\text { version }\end{array}$ & $\begin{array}{l}\text { Y } \\
\text { ES }\end{array}$ & $\begin{array}{l}\mathrm{N} \\
\mathrm{O}\end{array}$ & $\begin{array}{l}\mathrm{N} \\
\mathrm{O}\end{array}$ & $\begin{array}{l}\text { Y } \\
\text { ES }\end{array}$ & $\begin{array}{l}\mathrm{Y} \\
\mathrm{ES}\end{array}$ & $\begin{array}{l}\text { Y } \\
\text { ES }\end{array}$ & $\begin{array}{l}\text { Y } \\
\text { ES }\end{array}$ & $\begin{array}{l}\mathrm{Y} \\
\mathrm{ES}\end{array}$ & $\begin{array}{l}\mathrm{Y} \\
\mathrm{E} \\
\mathrm{S}\end{array}$ & $\begin{array}{l}\text { Y } \\
\text { ES }\end{array}$ & $\begin{array}{l}\text { Y } \\
\text { ES }\end{array}$ & $\begin{array}{l}\mathrm{Y} \\
\mathrm{ES}\end{array}$ & $\begin{array}{l}\text { Y } \\
\text { E } \\
\text { S }\end{array}$ & $\begin{array}{l}\mathrm{N} \\
\mathrm{O}\end{array}$ & $\begin{array}{l}\text { Y } \\
\text { ES }\end{array}$ & $\begin{array}{l}\text { Y } \\
\text { ES }\end{array}$ & $\begin{array}{l}\text { Y } \\
\text { ES }\end{array}$ \\
\hline $\begin{array}{l}\text { 7) } \\
\text { Event } \\
\text { calenda } \\
\mathbf{r} \\
\text { (update } \\
\text { d) }\end{array}$ & $\begin{array}{l}\text { Y } \\
\text { ES }\end{array}$ & $\begin{array}{l}\mathrm{Y} \\
\mathrm{ES}\end{array}$ & $\begin{array}{l}\text { Y } \\
\text { ES }\end{array}$ & $\begin{array}{l}\text { Y } \\
\text { ES }\end{array}$ & $\begin{array}{l}\text { Y } \\
\text { ES }\end{array}$ & $\begin{array}{l}\text { Y } \\
\text { ES }\end{array}$ & $\begin{array}{l}\text { Y } \\
\text { ES }\end{array}$ & $\begin{array}{l}\mathrm{Y} \\
\mathrm{ES}\end{array}$ & $\begin{array}{l}\text { Y } \\
\text { E } \\
\text { S }\end{array}$ & $\begin{array}{l}\text { Y } \\
\text { ES }\end{array}$ & $\begin{array}{l}\mathrm{Y} \\
\mathrm{ES}\end{array}$ & $\begin{array}{l}\mathrm{Y} \\
\mathrm{ES}\end{array}$ & $\begin{array}{l}\text { Y } \\
\text { E } \\
\text { S }\end{array}$ & $\begin{array}{l}\text { Y } \\
\text { ES }\end{array}$ & $\begin{array}{l}\text { Y } \\
\text { ES }\end{array}$ & $\begin{array}{l}\text { Y } \\
\text { ES }\end{array}$ & $\begin{array}{l}\text { Y } \\
\text { ES }\end{array}$ \\
\hline $\begin{array}{l}\text { 8) } \\
\text { Annual } \\
\text { financia } \\
\text { l } \\
\text { stateme } \\
\text { nts }\end{array}$ & $\begin{array}{l}\mathrm{Y} \\
\mathrm{ES}\end{array}$ & $\begin{array}{l}\mathrm{Y} \\
\mathrm{ES}\end{array}$ & $\begin{array}{l}\text { Y } \\
\text { ES }\end{array}$ & $\begin{array}{l}\text { Y } \\
\text { ES }\end{array}$ & $\begin{array}{l}\mathrm{Y} \\
\mathrm{ES}\end{array}$ & $\begin{array}{l}\text { Y } \\
\text { ES }\end{array}$ & $\begin{array}{l}\text { Y } \\
\text { ES }\end{array}$ & $\begin{array}{l}\mathrm{Y} \\
\mathrm{ES}\end{array}$ & $\begin{array}{l}\mathrm{N} \\
\mathrm{O}\end{array}$ & $\begin{array}{l}\text { Y } \\
\text { ES }\end{array}$ & $\begin{array}{l}\text { Y } \\
\text { ES }\end{array}$ & $\begin{array}{l}\mathrm{Y} \\
\mathrm{ES}\end{array}$ & $\begin{array}{l}\mathrm{N} \\
\mathrm{O}\end{array}$ & $\begin{array}{l}\text { Y } \\
\text { ES }\end{array}$ & $\begin{array}{l}\mathrm{Y} \\
\mathrm{ES}\end{array}$ & $\begin{array}{l}\text { Y } \\
\text { ES }\end{array}$ & $\begin{array}{l}\text { Y } \\
\text { ES }\end{array}$ \\
\hline $\begin{array}{l}\text { 9) } \\
\text { Indices }\end{array}$ & $\begin{array}{l}\mathrm{Y} \\
\mathrm{E} \\
\mathrm{S}\end{array}$ & \begin{tabular}{|l}
$\mathrm{Y}$ \\
$\mathrm{E}$ \\
$\mathrm{S}$
\end{tabular} & $\begin{array}{l}\mathrm{Y} \\
\mathrm{E} \\
\mathrm{S}\end{array}$ & \begin{tabular}{|l}
$\mathrm{Y}$ \\
$\mathrm{E}$ \\
$\mathrm{S}$
\end{tabular} & \begin{tabular}{|l}
$\mathrm{Y}$ \\
$\mathrm{E}$ \\
$\mathrm{S}$
\end{tabular} & $\begin{array}{l}\mathrm{Y} \\
\mathrm{E} \\
\mathrm{S}\end{array}$ & $\begin{array}{l}Y \\
E \\
S\end{array}$ & \begin{tabular}{|l}
$\mathrm{Y}$ \\
$\mathrm{E}$ \\
$\mathrm{S}$
\end{tabular} & $\begin{array}{l}\mathrm{N} \\
\mathrm{O}\end{array}$ & $\begin{array}{l}\mathrm{Y} \\
\mathrm{E} \\
\mathrm{S}\end{array}$ & \begin{tabular}{|l}
$\mathrm{Y}$ \\
$\mathrm{E}$ \\
$\mathrm{S}$
\end{tabular} & \begin{tabular}{|l}
$\mathrm{Y}$ \\
$\mathrm{E}$ \\
$\mathrm{S}$
\end{tabular} & $\begin{array}{l}\mathrm{N} \\
\mathrm{O}\end{array}$ & \begin{tabular}{|l}
$\mathrm{Y}$ \\
$\mathrm{E}$ \\
$\mathrm{S}$
\end{tabular} & \begin{tabular}{|l}
$\mathrm{Y}$ \\
$\mathrm{E}$ \\
$\mathrm{S}$
\end{tabular} & $\begin{array}{l}\mathrm{Y} \\
\mathrm{E} \\
\mathrm{S}\end{array}$ & $\begin{array}{l}Y \\
E \\
S\end{array}$ \\
\hline $\begin{array}{l}\text { 10) } \\
\text { Financi } \\
\text { al } \\
\text { stateme }\end{array}$ & \begin{tabular}{|l} 
Y \\
E \\
S
\end{tabular} & $\begin{array}{l}\mathrm{Y} \\
\mathrm{E} \\
\mathrm{S}\end{array}$ & $\begin{array}{l}\text { Y } \\
E \\
S\end{array}$ & \begin{tabular}{|l} 
Y \\
E \\
S
\end{tabular} & $\begin{array}{l}\mathrm{Y} \\
\mathrm{E} \\
\mathrm{S}\end{array}$ & $\begin{array}{l}\mathrm{Y} \\
\mathrm{E} \\
\mathrm{S}\end{array}$ & $\begin{array}{l}\mathrm{Y} \\
\mathrm{E} \\
\mathrm{S}\end{array}$ & $\begin{array}{l}\mathrm{Y} \\
\mathrm{E} \\
\mathrm{S}\end{array}$ & $\begin{array}{l}\mathrm{N} \\
\mathrm{O}\end{array}$ & $\begin{array}{l}\text { Y } \\
\text { E } \\
\text { S }\end{array}$ & \begin{tabular}{|l}
$\mathrm{Y}$ \\
$\mathrm{E}$ \\
$\mathrm{S}$
\end{tabular} & $\begin{array}{l}\mathrm{Y} \\
\mathrm{E} \\
\mathrm{S}\end{array}$ & $\begin{array}{l}\mathrm{N} \\
\mathrm{O}\end{array}$ & \begin{tabular}{|l}
$\mathrm{Y}$ \\
$\mathrm{E}$ \\
$\mathrm{S}$
\end{tabular} & $\begin{array}{l}\mathrm{Y} \\
\mathrm{E} \\
\mathrm{S}\end{array}$ & $\begin{array}{l}\mathrm{Y} \\
\mathrm{E} \\
\mathrm{S}\end{array}$ & $\begin{array}{l}\mathrm{Y} \\
\mathrm{E} \\
\mathrm{S}\end{array}$ \\
\hline
\end{tabular}




\begin{tabular}{|c|c|c|c|c|c|c|c|c|c|c|c|c|c|c|c|c|c|}
\hline $\begin{array}{l}\text { nt } \\
\text { archive }\end{array}$ & & & & & & & & & & & & & & & & & \\
\hline $\begin{array}{l}\text { PARTI } \\
\text { AL } \\
\text { INDEX }\end{array}$ & $\begin{array}{l}1 \\
0\end{array}$ & 8 & 8 & 8 & 9 & 9 & $\begin{array}{l}1 \\
0\end{array}$ & $\begin{array}{l}\mathbf{1} \\
\mathbf{0}\end{array}$ & 5 & 9 & $\begin{array}{l}\mathbf{1} \\
\mathbf{0}\end{array}$ & 9 & 6 & 6 & 9 & 9 & 9 \\
\hline
\end{tabular}

B) DISCLOSURE REGARDING THE ADMINISTRATIVE BODY AND

\begin{tabular}{|c|c|c|c|c|c|c|c|c|c|c|c|c|c|c|c|c|c|}
\hline \begin{tabular}{l|l}
$\mathbf{F}$ & \\
$\mathbf{E}$ & \\
$\mathbf{A}$ & $\mathbf{Z}$ \\
$\mathbf{T}$ & $\mathbf{Z}$ \\
$\mathbf{U}$ & 0 \\
$\mathbf{R}$ & $\mathbf{U}$ \\
$\mathbf{E}$ &
\end{tabular} & $\underset{\mathbb{4}}{4}$ & 孚 & 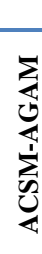 & $\frac{0}{2}$ & 至 & 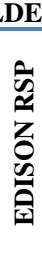 & 조도 & $\frac{\mathbb{x}}{x}$ & 荇 & 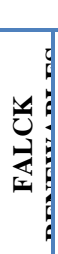 & $\frac{\mathbb{x}}{\underline{I}}$ & 罙 & 点 & 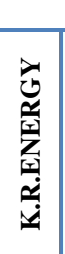 & $\sum_{i=1}^{\sum_{i}}$ & 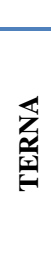 & 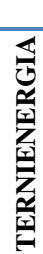 \\
\hline $\begin{array}{l}\text { 1) Members } \\
\text { of the } \\
\text { administrat } \\
\text { ive body }\end{array}$ & $\begin{array}{l}Y \\
E \\
S\end{array}$ & $\begin{array}{l}\text { Y } \\
\text { E } \\
\text { S }\end{array}$ & $\begin{array}{l}\text { Y } \\
\text { E } \\
\text { S }\end{array}$ & $\begin{array}{l}\text { Y } \\
\text { E } \\
\text { S }\end{array}$ & $\begin{array}{l}\mathrm{Y} \\
\mathrm{E} \\
\mathrm{S}\end{array}$ & $\begin{array}{l}Y \\
E \\
S\end{array}$ & $\begin{array}{l}\mathrm{Y} \\
\mathrm{F}\end{array}$ & $\begin{array}{l}\mathrm{Y} \\
\mathrm{E} \\
\mathrm{S}\end{array}$ & $\begin{array}{l}\text { Y } \\
\text { E } \\
\text { S }\end{array}$ & $\begin{array}{l}\text { Y } \\
\text { E } \\
\text { S }\end{array}$ & $\begin{array}{l}Y \\
E \\
S\end{array}$ & $\begin{array}{l}Y \\
E \\
S\end{array}$ & $\begin{array}{l}Y \\
E \\
S\end{array}$ & $\begin{array}{l}\mathrm{Y} \\
\mathrm{E} \\
\mathrm{S}\end{array}$ & $\begin{array}{l}\mathrm{Y} \\
\mathrm{E} \\
\mathrm{S}\end{array}$ & $\begin{array}{l}Y \\
E \\
S\end{array}$ & $\begin{array}{l}\mathrm{Y} \\
\mathrm{E} \\
\mathrm{S}\end{array}$ \\
\hline $\begin{array}{l}\text { 2) Structure } \\
\text { of the } \\
\text { administrat } \\
\text { ive body }\end{array}$ & $\begin{array}{l}\mathrm{Y} \\
\mathrm{E} \\
\mathrm{S}\end{array}$ & $\begin{array}{l}Y \\
\text { E } \\
\text { S }\end{array}$ & $\mathrm{Y}$ & $\begin{array}{l}\text { Y } \\
\mathrm{E}\end{array}$ & $\mathrm{Y}$ & Y & $\mathrm{Y}$ & $\mathrm{Y}$ & $\begin{array}{l}Y \\
\text { E } \\
S\end{array}$ & $\begin{array}{l}\text { Y } \\
\text { E } \\
\text { S }\end{array}$ & $\begin{array}{l}\mathrm{Y} \\
\mathrm{E} \\
\mathrm{S}\end{array}$ & $\begin{array}{l}\mathrm{Y} \\
\mathrm{E} \\
\mathrm{S}\end{array}$ & $\begin{array}{l}\mathrm{Y} \\
\mathrm{E} \\
\mathrm{S}\end{array}$ & $\begin{array}{l}\mathrm{Y} \\
\mathrm{E} \\
\mathrm{S}\end{array}$ & $\begin{array}{l}\mathrm{Y} \\
\mathrm{E} \\
\mathrm{S}\end{array}$ & $\begin{array}{l}\mathrm{Y} \\
\mathrm{E} \\
\mathrm{S}\end{array}$ & $\begin{array}{l}\mathrm{Y} \\
\mathrm{E} \\
\mathrm{S}\end{array}$ \\
\hline $\begin{array}{l}\text { 3) Curricula } \\
\text { of the } \\
\text { members of } \\
\text { the } \\
\text { administrat } \\
\text { ive body }\end{array}$ & $\begin{array}{l}\text { Y } \\
E \\
S\end{array}$ & $\begin{array}{l}\mathrm{Y} \\
\mathrm{E} \\
\mathrm{S}\end{array}$ & $\begin{array}{l}\mathrm{N} \\
\mathrm{O}\end{array}$ & $\begin{array}{l}\text { Y } \\
\text { E } \\
\text { S }\end{array}$ & $\begin{array}{l}\mathrm{Y} \\
\mathrm{E} \\
\mathrm{S}\end{array}$ & $\begin{array}{l}Y \\
E \\
S\end{array}$ & $\begin{array}{l}\mathrm{Y} \\
\mathrm{E} \\
\mathrm{S}\end{array}$ & $\begin{array}{l}\mathrm{Y} \\
\mathrm{E} \\
\mathrm{S}\end{array}$ & $\begin{array}{l}\text { Y } \\
\text { E } \\
\text { S }\end{array}$ & $\begin{array}{l}\mathrm{N} \\
\mathrm{O}\end{array}$ & $\begin{array}{l}\text { Y } \\
E \\
\text { S }\end{array}$ & $\begin{array}{l}Y \\
E \\
S\end{array}$ & $\begin{array}{l}Y \\
E \\
S\end{array}$ & $\begin{array}{l}\mathrm{Y} \\
\mathrm{E} \\
\mathrm{S}\end{array}$ & $\begin{array}{l}\mathrm{Y} \\
\mathrm{E} \\
\mathrm{S}\end{array}$ & $\begin{array}{l}\text { Y } \\
E \\
S\end{array}$ & $\begin{array}{l}\mathrm{N} \\
\mathrm{O}\end{array}$ \\
\hline $\begin{array}{l}\text { 4) } \\
\text { Functioning } \\
\text { of the } \\
\text { administrat } \\
\text { ive body }\end{array}$ & $\begin{array}{l}Y \\
E \\
S\end{array}$ & $\begin{array}{l}\mathrm{Y} \\
\mathrm{E} \\
\mathrm{S}\end{array}$ & $\mathrm{Y}$ & $S$ & $\begin{array}{l}\text { Y } \\
\text { E } \\
\text { S }\end{array}$ & S & $\mathrm{S}$ & $Y$ & $\begin{array}{l}\mathrm{N} \\
\mathrm{O}\end{array}$ & $\begin{array}{l}\mathrm{Y} \\
\mathrm{E} \\
\mathrm{S}\end{array}$ & $\begin{array}{l}\mathrm{Y} \\
\mathrm{E} \\
\mathrm{S}\end{array}$ & $\begin{array}{l}\mathrm{Y} \\
\mathrm{F}\end{array}$ & $\begin{array}{l}\mathrm{Y} \\
\mathrm{E} \\
\mathrm{S}\end{array}$ & $\mathrm{s}$ & $\begin{array}{l}\mathrm{Y} \\
\mathrm{E} \\
\mathrm{S}\end{array}$ & $\begin{array}{l}\text { Y } \\
\text { E } \\
\text { S }\end{array}$ & $\begin{array}{l}\mathrm{N} \\
\mathrm{O}\end{array}$ \\
\hline $\begin{array}{l}\text { 5) } \\
\text { Functioning } \\
\text { of } \\
\text { committees } \\
\text { within the } \\
\text { administrat } \\
\text { ive body }\end{array}$ & $\begin{array}{l}Y \\
E \\
S\end{array}$ & $\begin{array}{l}\mathrm{Y} \\
\mathrm{E} \\
\mathrm{S}\end{array}$ & $\begin{array}{l}\mathrm{N} \\
\mathrm{O}\end{array}$ & $\begin{array}{l}\text { Y } \\
\text { E } \\
\text { S }\end{array}$ & $\begin{array}{l}\mathrm{Y} \\
\mathrm{E} \\
\mathrm{S}\end{array}$ & S & S & $\mathrm{S}$ & $\begin{array}{l}\mathrm{N} \\
\mathrm{O}\end{array}$ & $\begin{array}{l}\mathrm{N} \\
\mathrm{O}\end{array}$ & $\begin{array}{l}Y \\
E \\
S\end{array}$ & $\begin{array}{l}\mathrm{N} \\
\mathrm{O}\end{array}$ & $\begin{array}{l}\mathrm{N} \\
\mathrm{O}\end{array}$ & $\begin{array}{l}\mathrm{Y} \\
\mathrm{E} \\
\mathrm{S}\end{array}$ & $\begin{array}{l}\mathrm{Y} \\
\mathrm{E} \\
\mathrm{S}\end{array}$ & $\begin{array}{l}Y \\
E \\
S\end{array}$ & $\begin{array}{l}\mathrm{N} \\
\mathrm{O}\end{array}$ \\
\hline $\begin{array}{l}\text { 6) } \\
\text { Remunerati } \\
\text { on policies }\end{array}$ & $\begin{array}{l}\mathrm{Y} \\
\mathrm{E} \\
\mathrm{S}\end{array}$ & I & $\begin{array}{l}\mathrm{N} \\
\mathrm{O}\end{array}$ & Y & $\begin{array}{l}\mathrm{E} \\
\mathrm{S}\end{array}$ & $\mathrm{s}$ & & & $\begin{array}{l}\mathrm{N} \\
\mathrm{O}\end{array}$ & $\mathrm{O}$ & $\begin{array}{l}\mathrm{E} \\
\mathrm{S}\end{array}$ & $\begin{array}{l}\mathrm{N} \\
\mathrm{O}\end{array}$ & $\begin{array}{l}\text { Y } \\
\text { E } \\
\text { S }\end{array}$ & $\begin{array}{l}\mathrm{Y} \\
\mathrm{E} \\
\mathrm{S}\end{array}$ & $\begin{array}{l}\mathrm{Y} \\
\mathrm{E} \\
\mathrm{S}\end{array}$ & $\begin{array}{l}\text { Y } \\
\text { E } \\
\text { S }\end{array}$ & $\begin{array}{l}\text { Y } \\
\text { E } \\
\text { S }\end{array}$ \\
\hline $\begin{array}{l}\text { 7) } \\
\text { Emolument } \\
\text { s of the } \\
\text { administrat } \\
\text { ive body } \\
\text { members }\end{array}$ & $\begin{array}{l}\mathrm{N} \\
\mathrm{O}\end{array}$ & $\begin{array}{l}\mathrm{N} \\
\mathrm{O}\end{array}$ & $\begin{array}{l}\mathrm{N} \\
\mathrm{O}\end{array}$ & $\begin{array}{l}\mathrm{N} \\
\mathrm{O}\end{array}$ & $\begin{array}{l}\mathrm{Y} \\
\mathrm{E} \\
\mathrm{S}\end{array}$ & $\begin{array}{l}Y \\
E \\
S\end{array}$ & $\begin{array}{l}\mathrm{Y} \\
\mathrm{E} \\
\mathrm{S}\end{array}$ & $\begin{array}{l}\mathrm{Y} \\
\mathrm{E} \\
\mathrm{S}\end{array}$ & $\begin{array}{l}\mathrm{N} \\
\mathrm{O}\end{array}$ & $\begin{array}{l}\mathrm{N} \\
\mathrm{O}\end{array}$ & $\begin{array}{l}\text { Y } \\
E \\
\text { S }\end{array}$ & $\begin{array}{l}\mathrm{N} \\
\mathrm{O}\end{array}$ & $\begin{array}{l}\mathrm{N} \\
\mathrm{O}\end{array}$ & $\begin{array}{l}\mathrm{Y} \\
\mathrm{E} \\
\mathrm{S}\end{array}$ & $\begin{array}{l}\mathrm{Y} \\
\mathrm{E} \\
\mathrm{S}\end{array}$ & $\begin{array}{l}\text { Y } \\
E \\
\text { S }\end{array}$ & $\begin{array}{l}\mathrm{N} \\
\mathrm{O}\end{array}$ \\
\hline $\begin{array}{l}\text { 8) Election } \\
\text { procedure }\end{array}$ & $\begin{array}{l}\mathrm{Y} \\
\mathrm{E} \\
\mathrm{S}\end{array}$ & $\begin{array}{l}Y \\
E \\
S\end{array}$ & $\mathbf{r}$ & $\mathrm{Y}$ & $\begin{array}{l}\mathrm{Y} \\
\mathrm{E} \\
\mathrm{S}\end{array}$ & $\begin{array}{l}\mathrm{Y} \\
\mathrm{E} \\
\mathrm{S}\end{array}$ & $\mathbf{r}$ & $\mathrm{Y}$ & $\begin{array}{l}\mathrm{N} \\
\mathrm{O}\end{array}$ & $\begin{array}{l}\mathrm{Y} \\
\mathrm{E} \\
\mathrm{S}\end{array}$ & $\begin{array}{l}\text { Y } \\
\text { E } \\
\text { S }\end{array}$ & $\begin{array}{l}\text { Y } \\
\text { E } \\
\text { S }\end{array}$ & $\begin{array}{l}\text { Y } \\
\text { E } \\
\text { S }\end{array}$ & $\begin{array}{l}\mathrm{Y} \\
\mathrm{E} \\
\mathrm{S}\end{array}$ & $\begin{array}{l}\mathrm{Y} \\
\mathrm{E} \\
\mathrm{S}\end{array}$ & $\begin{array}{l}\text { Y } \\
\text { E } \\
\text { S }\end{array}$ & $\begin{array}{l}\mathrm{Y} \\
\mathrm{E} \\
\mathrm{S}\end{array}$ \\
\hline $\begin{array}{l}\text { 9) Major } \\
\text { shareholder } \\
\text { s }\end{array}$ & $\begin{array}{l}\mathrm{Y} \\
\mathrm{E} \\
\mathrm{S}\end{array}$ & $\begin{array}{l}\mathrm{Y} \\
\mathrm{E} \\
\mathrm{S}\end{array}$ & $\begin{array}{l}\mathrm{Y} \\
\mathrm{E}\end{array}$ & $\mathrm{Y}$ & $\mathrm{Y}$ & $\begin{array}{l}\mathrm{Y} \\
\mathrm{E} \\
\mathrm{S}\end{array}$ & $\mathrm{Y}$ & 1 & $\mathrm{O}$ & $\begin{array}{l}\mathrm{Y} \\
\mathrm{E} \\
\mathrm{S}\end{array}$ & $\begin{array}{l}\text { Y } \\
\text { E } \\
\text { S }\end{array}$ & $\begin{array}{l}\text { Y } \\
\text { E } \\
\text { S }\end{array}$ & $\begin{array}{l}\text { Y } \\
\text { E } \\
\text { S }\end{array}$ & $\begin{array}{l}\mathrm{Y} \\
\mathrm{E} \\
\mathrm{S}\end{array}$ & $\begin{array}{l}Y \\
E \\
S\end{array}$ & $\begin{array}{l}\mathrm{Y} \\
\mathrm{E} \\
\mathrm{S}\end{array}$ & $\begin{array}{l}\mathrm{Y} \\
\mathrm{E} \\
\mathrm{S}\end{array}$ \\
\hline
\end{tabular}




\begin{tabular}{|c|c|c|c|c|c|c|c|c|c|c|c|c|c|c|c|c|c|}
\hline $\begin{array}{l}\text { 10) Share } \\
\text { ownership } \\
\text { of } \\
\text { administrat } \\
\text { ive body } \\
\text { members }\end{array}$ & $\begin{array}{l}\mathrm{Y} \\
\mathrm{E} \\
\mathrm{S}\end{array}$ & $\begin{array}{l}Y \\
E \\
S\end{array}$ & $\begin{array}{l}\mathrm{Y} \\
\mathrm{E} \\
\mathrm{S}\end{array}$ & $\begin{array}{l}Y \\
E \\
S\end{array}$ & $\begin{array}{l}Y \\
E \\
S\end{array}$ & $\begin{array}{l}Y \\
E \\
S\end{array}$ & $\begin{array}{l}Y \\
E \\
S\end{array}$ & $\begin{array}{l}Y \\
E \\
S\end{array}$ & $\begin{array}{l}\mathrm{N} \\
\mathrm{O}\end{array}$ & $\begin{array}{l}\mathrm{N} \\
\mathrm{O}\end{array}$ & $\begin{array}{l}Y \\
E \\
S\end{array}$ & $\begin{array}{l}Y \\
E \\
S\end{array}$ & $\begin{array}{l}Y \\
E \\
S\end{array}$ & $\begin{array}{l}Y \\
E \\
S\end{array}$ & $\begin{array}{l}\mathrm{N} \\
\mathrm{O}\end{array}$ & $\begin{array}{l}\mathrm{N} \\
\mathrm{O}\end{array}$ & $\begin{array}{l}\text { Y } \\
\text { E } \\
\text { S }\end{array}$ \\
\hline & & & & & & & & & & & & & & & & & \\
\hline $\begin{array}{l}\text { PARTIAL } \\
\text { INDEX }\end{array}$ & 9 & 9 & 7 & 9 & 10 & 10 & 10 & 10 & 3 & 5 & 10 & 7 & 8 & 10 & 9 & 9 & 6 \\
\hline \multicolumn{18}{|c|}{$\begin{array}{l}\text { C) DISCLOSURE ON OTHER ELEMENTS OF CORPORATE } \\
\text { GOVERNANCE }\end{array}$} \\
\hline \begin{tabular}{l|l}
$\mathbf{F}$ & \\
$\mathbf{E}$ & $\mathbf{Z}$ \\
$\mathbf{A}$ & $\mathbf{Z}$ \\
$\mathbf{T}$ & $\mathbf{Z}$ \\
$\mathbf{U}$ & 0 \\
$\mathbf{R}$ & $\circlearrowright$ \\
$\mathbf{E}$ & \\
\end{tabular} & $\underset{\nwarrow}{\gtrless}$ & 空 & 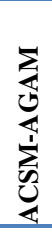 & 乙豆: & 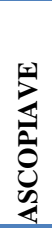 & 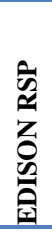 & 谷 & \begin{tabular}{l}
0 \\
\multirow{x}{x}{} \\
\end{tabular} & 氙 & 过 & $\underset{\underline{x}}{\mathbb{x}}$ & 公 & 占 & 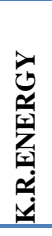 & 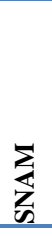 & 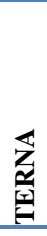 & 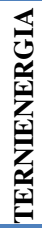 \\
\hline $\begin{array}{l}\text { 1) Rules } \\
\text { and } \\
\text { procedure } \\
\text { s }\end{array}$ & $\begin{array}{l}\mathrm{N} \\
\mathrm{O}\end{array}$ & $\begin{array}{l}\mathrm{Y} \\
\mathrm{E} \\
\mathrm{S}\end{array}$ & $\begin{array}{l}\mathrm{Y} \\
\mathrm{E} \\
\mathrm{S}\end{array}$ & $\begin{array}{l}\mathrm{Y} \\
\mathrm{E} \\
\mathrm{S}\end{array}$ & $\begin{array}{l}Y \\
E \\
S\end{array}$ & $\begin{array}{l}\mathrm{Y} \\
\mathrm{E} \\
\mathrm{S}\end{array}$ & $\begin{array}{l}\mathrm{Y} \\
\mathrm{E} \\
\mathrm{S}\end{array}$ & $\begin{array}{l}Y \\
E \\
S\end{array}$ & $\begin{array}{l}\mathrm{N} \\
\mathrm{O}\end{array}$ & $\begin{array}{l}\mathrm{N} \\
\mathrm{O}\end{array}$ & $\begin{array}{l}Y \\
E \\
S\end{array}$ & $\begin{array}{l}Y \\
E \\
S\end{array}$ & $\begin{array}{l}\mathrm{Y} \\
\mathrm{E} \\
\mathrm{S}\end{array}$ & $\begin{array}{l}Y \\
E \\
S\end{array}$ & $\begin{array}{l}Y \\
E \\
S\end{array}$ & $\begin{array}{l}Y \\
E \\
S\end{array}$ & $\begin{array}{l}\mathrm{N} \\
\mathrm{O}\end{array}$ \\
\hline $\begin{array}{l}\text { 2) } \\
\text { Company } \\
\text { statute }\end{array}$ & $\begin{array}{l}\mathrm{Y} \\
\mathrm{E} \\
\mathrm{S}\end{array}$ & $\begin{array}{l}Y \\
E \\
S\end{array}$ & $\begin{array}{l}\mathrm{Y} \\
\mathrm{E} \\
\mathrm{S}\end{array}$ & $\begin{array}{l}Y \\
E \\
S\end{array}$ & $\begin{array}{l}\mathrm{Y} \\
\mathrm{E} \\
\mathrm{S}\end{array}$ & $\begin{array}{l}Y \\
E \\
S\end{array}$ & $\begin{array}{l}Y \\
E \\
S\end{array}$ & $\begin{array}{l}\mathrm{Y} \\
\mathrm{E} \\
\mathrm{S}\end{array}$ & $\begin{array}{l}\mathrm{N} \\
\mathrm{O}\end{array}$ & $\begin{array}{l}\mathrm{N} \\
\mathrm{O}\end{array}$ & $\begin{array}{l}Y \\
E \\
S\end{array}$ & $\begin{array}{l}Y \\
E \\
S\end{array}$ & $\begin{array}{l}\mathrm{Y} \\
\mathrm{E} \\
\mathrm{S} \\
\end{array}$ & $\begin{array}{l}Y \\
E \\
S\end{array}$ & $\begin{array}{l}\text { Y } \\
E \\
S\end{array}$ & $\begin{array}{l}Y \\
\text { E } \\
\text { S }\end{array}$ & $\begin{array}{l}\mathrm{N} \\
\mathrm{O}\end{array}$ \\
\hline $\begin{array}{l}\text { 3) Ethical } \\
\text { code }\end{array}$ & $\begin{array}{l}\mathrm{Y} \\
\mathrm{E} \\
\mathrm{S}\end{array}$ & $\begin{array}{l}\text { Y } \\
\text { E } \\
\text { S }\end{array}$ & $\begin{array}{l}\mathrm{Y} \\
\mathrm{E} \\
\mathrm{S}\end{array}$ & $\begin{array}{l}\mathrm{Y} \\
\mathrm{E} \\
\mathrm{S}\end{array}$ & $\begin{array}{l}\mathrm{Y} \\
\mathrm{E} \\
\mathrm{S}\end{array}$ & $\begin{array}{l}\mathrm{Y} \\
\mathrm{E} \\
\mathrm{S}\end{array}$ & $\begin{array}{l}\mathrm{Y} \\
\mathrm{E} \\
\mathrm{S}\end{array}$ & $\begin{array}{l}\mathrm{Y} \\
\mathrm{E} \\
\mathrm{S}\end{array}$ & $\begin{array}{l}\mathrm{N} \\
\mathrm{O}\end{array}$ & $\begin{array}{l}\mathrm{N} \\
\mathrm{O}\end{array}$ & $\begin{array}{l}\text { Y } \\
\text { E } \\
S\end{array}$ & $\begin{array}{l}\text { Y } \\
\text { E } \\
S\end{array}$ & $\begin{array}{l}\mathrm{Y} \\
\mathrm{E} \\
\mathrm{S}\end{array}$ & $\begin{array}{l}\text { Y } \\
E \\
S\end{array}$ & $\begin{array}{l}\text { Y } \\
E \\
S\end{array}$ & $\begin{array}{l}Y \\
\text { E } \\
\text { S }\end{array}$ & $\begin{array}{l}\mathrm{N} \\
\mathrm{O}\end{array}$ \\
\hline $\begin{array}{l}\text { 4) } \\
\text { Sustainab } \\
\text { ility } \\
\text { report }\end{array}$ & $\begin{array}{l}\mathrm{Y} \\
\mathrm{E} \\
\mathrm{S}\end{array}$ & $\begin{array}{l}\mathrm{Y} \\
\mathrm{E} \\
\mathrm{S}\end{array}$ & $\begin{array}{l}\mathrm{N} \\
\mathrm{O}\end{array}$ & $\begin{array}{l}\mathrm{N} \\
\mathrm{O}\end{array}$ & $\begin{array}{l}\mathrm{N} \\
\mathrm{O}\end{array}$ & $\begin{array}{l}\mathrm{Y} \\
\mathrm{E} \\
\mathrm{S}\end{array}$ & $\begin{array}{l}\mathrm{Y} \\
\mathrm{E} \\
\mathrm{S}\end{array}$ & $\begin{array}{l}\mathrm{Y} \\
\mathrm{E} \\
\mathrm{S}\end{array}$ & $\begin{array}{l}\mathrm{N} \\
\mathrm{O}\end{array}$ & $\begin{array}{l}\mathrm{N} \\
\mathrm{O}\end{array}$ & $\begin{array}{l}Y \\
E \\
S\end{array}$ & $\begin{array}{l}Y \\
E \\
S\end{array}$ & $\begin{array}{l}Y \\
E \\
S\end{array}$ & $\begin{array}{l}\mathrm{N} \\
\mathrm{O}\end{array}$ & $\begin{array}{l}Y \\
E \\
S\end{array}$ & $\begin{array}{l}Y \\
E \\
S\end{array}$ & $\begin{array}{l}\mathrm{N} \\
\mathrm{O}\end{array}$ \\
\hline $\begin{array}{l}\text { 5) } \\
\text { Informati } \\
\text { on on the } \\
\text { board of } \\
\text { statutory } \\
\text { auditors }\end{array}$ & $\begin{array}{l}\mathrm{Y} \\
\mathrm{E} \\
\mathrm{S}\end{array}$ & $\begin{array}{l}Y \\
E \\
S\end{array}$ & $\begin{array}{l}Y \\
E \\
S\end{array}$ & $\begin{array}{l}Y \\
E \\
S\end{array}$ & $\begin{array}{l}Y \\
E \\
S\end{array}$ & $\begin{array}{l}Y \\
E \\
S\end{array}$ & $\begin{array}{l}Y \\
E \\
S\end{array}$ & $\begin{array}{l}\mathrm{Y} \\
\mathrm{E} \\
\mathrm{S}\end{array}$ & $\begin{array}{l}Y \\
E \\
S\end{array}$ & $\begin{array}{l}Y \\
E \\
S\end{array}$ & $\begin{array}{l}Y \\
E \\
S\end{array}$ & $\begin{array}{l}Y \\
E \\
S\end{array}$ & $\begin{array}{l}\mathrm{Y} \\
\mathrm{E} \\
\mathrm{S}\end{array}$ & $\begin{array}{l}Y \\
E \\
S\end{array}$ & $\begin{array}{l}Y \\
E \\
S\end{array}$ & $\begin{array}{l}\text { Y } \\
\text { E } \\
\text { S }\end{array}$ & $\begin{array}{l}Y \\
E \\
S\end{array}$ \\
\hline $\begin{array}{l}\text { 6) Analyst } \\
\text { coverage }\end{array}$ & $\begin{array}{l}Y \\
E \\
S\end{array}$ & $\begin{array}{l}Y \\
E \\
S\end{array}$ & $\begin{array}{l}\mathrm{N} \\
\mathrm{O}\end{array}$ & $\begin{array}{l}\mathrm{N} \\
\mathrm{O}\end{array}$ & $\begin{array}{l}\mathrm{N} \\
\mathrm{O}\end{array}$ & $\begin{array}{l}Y \\
E \\
S\end{array}$ & $\begin{array}{l}Y \\
E \\
S\end{array}$ & $\begin{array}{l}\mathrm{Y} \\
\mathrm{E} \\
\mathrm{S}\end{array}$ & $\begin{array}{l}\mathrm{N} \\
\mathrm{O}\end{array}$ & $\begin{array}{l}Y \\
E \\
S\end{array}$ & $\begin{array}{l}Y \\
E \\
S\end{array}$ & $\begin{array}{l}Y \\
E \\
S\end{array}$ & $\begin{array}{l}\mathrm{Y} \\
\mathrm{E} \\
\mathrm{S} \\
\end{array}$ & $\begin{array}{l}\mathrm{N} \\
\mathrm{O}\end{array}$ & $\begin{array}{l}Y \\
E \\
S\end{array}$ & $\begin{array}{l}Y \\
E \\
S\end{array}$ & $\begin{array}{l}\mathrm{N} \\
\mathrm{O}\end{array}$ \\
\hline $\begin{array}{l}\text { 7) } \\
\text { Auditing } \\
\text { firm }\end{array}$ & $\begin{array}{l}\mathrm{N} \\
\mathrm{O}\end{array}$ & $\begin{array}{l}\mathrm{N} \\
\mathrm{O}\end{array}$ & $\begin{array}{l}\mathrm{N} \\
\mathrm{O}\end{array}$ & $\begin{array}{l}\mathrm{N} \\
\mathrm{O}\end{array}$ & $\begin{array}{l}\mathrm{Y} \\
\mathrm{E} \\
\mathrm{S}\end{array}$ & $\begin{array}{l}Y \\
E \\
S\end{array}$ & $\begin{array}{l}Y \\
E \\
S\end{array}$ & $\begin{array}{l}Y \\
E \\
S\end{array}$ & $\begin{array}{l}\mathrm{N} \\
\mathrm{O}\end{array}$ & $\begin{array}{l}\mathrm{N} \\
\mathrm{O}\end{array}$ & $\begin{array}{l}Y \\
E \\
S\end{array}$ & $\begin{array}{l}Y \\
E \\
S\end{array}$ & $\begin{array}{l}\mathrm{Y} \\
\mathrm{E} \\
\mathrm{S}\end{array}$ & $\begin{array}{l}\text { Y } \\
\text { E } \\
S\end{array}$ & $\begin{array}{l}\text { Y } \\
\text { E } \\
S\end{array}$ & $\begin{array}{l}\text { Y } \\
\text { E } \\
\text { S }\end{array}$ & $\begin{array}{l}\text { Y } \\
\text { E } \\
\text { S }\end{array}$ \\
\hline $\begin{array}{l}\text { PARTIA } \\
\text { L INDEX }\end{array}$ & $\begin{array}{l}7, \\
14\end{array}$ & $\begin{array}{l}\mathbf{8} \\
\mathbf{5 7}\end{array}$ & $\begin{array}{l}5, \\
71\end{array}$ & $\begin{array}{l}5, \\
71\end{array}$ & $\begin{array}{l}7, \\
14\end{array}$ & 10 & 10 & 10 & $\begin{array}{l}1, \\
43\end{array}$ & $\begin{array}{l}2, \\
86\end{array}$ & 10 & 10 & 10 & $\begin{array}{l}7, \\
14\end{array}$ & 10 & 10 & $\begin{array}{l}2, \\
86\end{array}$ \\
\hline
\end{tabular}

Source: (own processing) 
Table E - Results obtained

\begin{tabular}{|c|c|c|c|c|}
\hline COMPANY NAME & $\begin{array}{c}\text { A) } \\
\text { WEBSITE } \\
\text { USABILIT } \\
\mathbf{Y}\end{array}$ & $\begin{array}{c}\text { B) } \\
\text { DISCLOSURE } \\
\text { REGARDING THE } \\
\text { ADMINISTRATIV } \\
\text { E BODY AND } \\
\text { SHAREHOLDERS }\end{array}$ & $\begin{array}{c}\text { C) } \\
\text { DISCLOSURE } \\
\text { ON OTHER } \\
\text { ELEMENTS } \\
\text { OF } \\
\text { CORPORATE } \\
\text { GOVERNANC } \\
\text { E }\end{array}$ & $\begin{array}{c}\text { TOTA } \\
\text { L } \\
\text { INDEX }\end{array}$ \\
\hline A2A & 10 & 9 & 7,14 & 8,71 \\
\hline ACEA & 8 & 9 & 8,57 & 8,52 \\
\hline ACSM-AGAM & 8 & 7 & 5,71 & 6,90 \\
\hline $\begin{array}{l}\text { ALERION } \\
\text { CLEANPOWER }\end{array}$ & 8 & 9 & 5,71 & $\mathbf{7 , 5 7}$ \\
\hline ASCOPIAVE & 9 & 10 & 7,14 & 8,71 \\
\hline EDISON RSP & 9 & 10 & 10 & 9,67 \\
\hline ENEL & 10 & 10 & 10 & 10,00 \\
\hline ERG & 10 & 10 & 10 & 10,00 \\
\hline ERGYCAPITAL & 5 & 3 & 1,43 & 3,14 \\
\hline FALCK RENEWABLES & 9 & 5 & 2,86 & 5,62 \\
\hline HERA & 10 & 10 & 10 & 10 \\
\hline IREN & 9 & 7 & 10 & 8,67 \\
\hline ITALGAS & 6 & 8 & 10 & 8,00 \\
\hline K.R.ENERGY & 6 & 10 & 7,14 & 7,71 \\
\hline SNAM & 9 & 9 & 10 & $\mathbf{9 , 3 3}$ \\
\hline TERNA & 9 & 9 & 10 & 9,33 \\
\hline TERNIENERGIA & 9 & 6 & 2,86 & 5,95 \\
\hline AVERAGE & 8,47 & 8,29 & 7,56 & 8,11 \\
\hline MAXIMUM & 10 & 10 & 10 & 10,00 \\
\hline MINIMUM & 5 & 3 & 1,43 & 3,14 \\
\hline
\end{tabular}

Source: (own processing)

\section{Conclusion:}

Interesting results emerged from the analysis of disclosure on the websites of companies in the FTSE Italy Public Services sector.

Regarding site usability and disclosure of financial information online, 11 companies provide a level of disclosure above the average (A2a, Ascopiave, Edison Rsp, Enel, Erg, Falck Renewables, Hera, Iren, Snam, Terna and TerniEnergia). The companies with greater disclosure are A2a, Enel, Erg and Hera with a partial index value of 10, while the company Ergycapital is the company that obtained the lowest index value of 5.

Eleven companies provide disclosure regarding the board of directors and shareholders that is greater than the average (A2a, Acea, Alerion CleanPower, Ascopiave, Edison Rsp, Enel, Erg, Hera, K.R.Energy, Snam, Terna). The companies that take the greater disclosure are Ascopiave, Enel, Edison Rsp, Enel, Erg, Hera and K.R.Energy with an index value of 10. In 
other words, such companies possess all the considered characteristics. The company with the lowest index value is Ergycapital with a score of 3.

As for disclosure regarding other elements of corporate governance, 9 companies obtain an index value greater than the average (Acea, Edison Rsp, Enel, Erg, Hera, Iren, Italgas, Snam and Terna). It is necessary to highlight that the maximum value of this partial index is achieved by 3 companies (Enel, Erg and Hera) while the minimum value is achieved by 1 company (Ergycapital).

With regards to the total disclosure index, 10 companies obtain a value higher than that of the sample average (A2a, Acea, Ascopiave, Edison Rsp, Enel, Erg, Hera, Iren, Snam and Terna); the maximum value is achieved by Enel, Erg and Hera (10,00) and the lowest value by Ergycapital.

Based on the variables considered in the analysis of the websites, no company provides total disclosure with maximum values. The company which offers total disclosure with the highest value is Enel (9.52) while Ergycapital has the lowest value (3.14).

Based on the newly exposed data, we can certainly say that, apart from a few companies, it is necessary to improve the quality of disclosure of the public utilities companies listed on the Italian Stock Exchange.

\section{References:}

1. Badia F., (2009), Sistemi e strumenti di corporate governance nelle local utilities, FrancoAngeli, Milano.

2. Bassi G., (2012), I servizi pubblici locali di rilevanza economica, Maggioli, Bologna.

3. Bianchi M., Ciavarella A., Novembre V., Signoretti R., (2011), Comply or explain: Investor protection through the Italian corporate governance code, Journal of Applied Corporate Finance, 23, 107-121.

4. Bonson E., Escobar T., (2006), Digital reporting in Eastern Europe: An empirical study, International Journal of Accounting Information Systems, 7(4), 299-318.

5. Chan M. C., Watson J., (2011), Voluntary disclosure of segment information in a regulated environment: Australian evidence, Eurasian Business Review, 1(1), 37-53.

6. Cheffins, B., (2012) The history of corporate governance, ECGI Working Paper Series in Law, 184, 1-38.

7. Craven B. M., Marston C. L., (1999), Financial reporting on the internet by leading UK companies, The European Accounting Review, 8(2), 321-333.

8. Cucuzza T. G., Cherian J., (2001), The internet e-business: Trends and implications for the finance function, Journal of Cost Management, 15(3), 5-14. 
9. Debreceny R., Rahman A., (2005), Firm-specific determinants of continuous corporate disclosures, The International Journal of Accounting, 40(3), 249-278.

10. Debreceny R., Glen L., Rahman A., (2002), The determinants of internet financial reporting, Journal of Accounting and Public Policy, 21(4), 371-394.

11. Gandia J. L., (2008), Determinants of internet-based corporate governance disclosure by Spanish listed companies, Online Information Review, 32(6), 791-817.

12. Glaum M., Schmidt P., Street D., Vogel S., (2013), Compliance with IFRS 3 and IAS 36 required disclosures across 17 European countries: Company and country level determinants, Accounting and Business Research, 43(3), 163-204.

13. Kahn T., (2007), Internet financial reporting: Disclosure about companies on websites, Journal Business Systems, Governance and Ethics, 2(2), 37-46.

14. Liberatore G., (2001), Pianificazione e controllo delle aziende di trasporto pubblico locale: problematiche di misurazione della performance, FrancoAngeli, Milano.

15. Liguori F., (2007), I servizi pubblici locali, Giappichelli, Torino.

16. Lostorto V., (2008), I servizi pubblici. Il quadro normativo, organizzazione, i modelli gestionali, FrancoAngeli, Milano.

17. Maronti G., Iuliano G., (2012), Voluntary adoption of IFRS by Italian private firms: A study of the determinants, Eurasian Business Review, 2(2), 43-70.

18. Quagli A., (2002), Internet e la comunicazione finanziaria, Milano, FrancoAngeli.

19. Rizzo G., (2012), La concessione di servizi, Giappichelli, Torino.

20. Rizzoni S., (2008), La gestione dei servizi pubblici. Da aziende speciali a società in house, FrancoAngeli, Milano.

21. Salomone R., Franco G., (2006), Dalla qualità totale alla qualità integrata. L'integrazione dei sistemi di gestione qualità, ambiente, sicurezza ed etica per il vantaggio competitivo, FrancoAngeli, Milano.

22. Stiglitz J. E., (2003), Economia del settore pubblico, Milano, Hoepli.

23. Trimarchi F., (1999), Le società miste per i servizi locali, Giuffrè, Milano.

24. Vigneri A., De Vincenti C., (2011), I servizi pubblici locali tra riforma e referendum, Maggioli, Bologna.

25. Xiao Z., Yang H., Chow C., (2004), Patterns and determinants of internet based corporate disclosure in China, Journal of Accounting and Public Policy, 23(3), 191-225. 